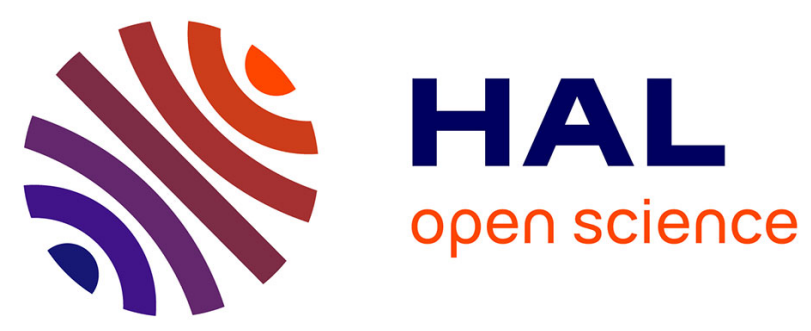

\title{
MISFET-based biosensing interface for neurons guided growth and neuronal electrical activities recording
}

Florian Larramendy, Amel Bendali, Marie-Charline Blatché, Fabrice Mathieu, Serge Picaud, Pierre Temple-Boyer, Liviu Nicu

\section{- To cite this version:}

Florian Larramendy, Amel Bendali, Marie-Charline Blatché, Fabrice Mathieu, Serge Picaud, et al.. MISFET-based biosensing interface for neurons guided growth and neuronal electrical activities recording. Sensors and Actuators B: Chemical, 2014, 203, pp.375 - 381. 10.1016/j.snb.2014.06.106 . hal01504965

\section{HAL Id: hal-01504965 \\ https://hal.science/hal-01504965}

Submitted on 10 Apr 2017

HAL is a multi-disciplinary open access archive for the deposit and dissemination of scientific research documents, whether they are published or not. The documents may come from teaching and research institutions in France or abroad, or from public or private research centers.
L'archive ouverte pluridisciplinaire HAL, est destinée au dépôt et à la diffusion de documents scientifiques de niveau recherche, publiés ou non, émanant des établissements d'enseignement et de recherche français ou étrangers, des laboratoires publics ou privés. 


\title{
MISFET-based biosensing interface for neurons guided growth and neuronal electrical activities recording
}

\author{
F. Larramendy ${ }^{1,2}$, A. Bendali3 ${ }^{3,4,5}$, M.C. Blatché ${ }^{1,2}$, F. Mathieu ${ }^{1,2}$, \\ S. Picaud ${ }^{3,4,5,6}$, P. Temple-Boyer ${ }^{1,2}, \underline{\text { L. Nicu }}^{1,2}$ \\ ${ }^{1}$ CNRS, LAAS, 7 avenue du colonel Roche, F-31400 Toulouse, France \\ 2 University of Toulouse, UPS, LAAS, F-31400 Toulouse, France \\ ${ }^{3}$ INSERM, U968, Institut de la Vision, Paris, 75012, France \\ ${ }^{4}$ UPMC, University of Paris 06, UMRS968, Institut de la Vision, Paris, 75012, France \\ ${ }^{5}$ CNRS, UMR7210, Institut de la Vision, Paris, 75012, France \\ ${ }^{6}$ Fondation Ophtalmologique Adolphe de Rothschild, Paris, 75019, France
}

\begin{abstract}
A hybrid circuit of a transistor-based chip was implemented and characterized for the neuronal electrical activity recording. The integration of microfluidic architectures was developed to control precisely neurites outgrowth and form topologically defined and stable neural networks. Individual neural cells from rat retinae and Lymnaea stagnalis snails were immobilized on gates regions of Metal Insulator Semiconductor Field Effect Transistors (MISFET). Neuronal orientation was achieved in both cases but neuronal action potentials were only recorded in the Lymnaea stagnalis case. They were successfully triggered and inhibited by implementing a picrotoxin GABA - picrotoxin injection protocol, exhibiting a direct influence of picrotoxin on the "spike type" action potential waveform. The implementation of the whole process of neuronal culture and subsequent activity monitoring constitutes a proof-of-principle experiment for the development of neuroelectronic systems for signal processing studies adapted to low-density neuronal cultures.
\end{abstract}

Keywords: FET microdevice, neuronal interface, action potential measurement 


\section{Introduction}

The study of the neuronal network dynamics in brain tissue is a difficult task owing to the large number of closely packed neurons with complex synaptic connectivity and the difficulty of monitoring activity in the neural network at the single-cell resolution with non-invasive techniques. In principle, low-density cultures of nerve cells drastically reduce the network complexity but uncertainty on the synaptic wiring remains. Furthermore, while single cell recording can be easily obtained by patch clamp recording [1], it is very challenging to achieve multicellular recordings with this approach. By contrast, the use of cell non-invasive extracellular multi-electrode array (MEA) enabled recordings and stimulations of large cell numbers [2,3]. Similarly, planar MEA were used for neurons culture for days and months without inflicting mechanical damage to the neuronal membrane [4]. This involves electrical measurements (voltage or current) across the cell membrane [5]. Nevertheless, such extracellular electrical interfacing of neurons was implemented with planar metal electrodes on insulating substrates [6-8], associated with organic transistors [9] or integrated with semiconductor devices using Complementary Metal Oxide Semiconductor (CMOS) technology $[10,11]$. In all cases, the activity of a nerve cell - i.e. the action potential - is stimulated and/or recorded by interfacing individual nerve cells with electrical microdevices that locally generate and/or sense electrical field variations towards medical applications [12]. So far, efforts have been focused onto interfacing nerve cells in order to measure and interpret the neuronal action potential and greater sensitivity would be required to measure smaller cell signals. One of the first way to "communicate" with nerve cells or to make them inter-communicate using microelectrodes was achieved by Jerome Pine [13] and Guenter Gross [14]. Then, Peter Fromherz proposed to use MOS technology in order to integrate microelectrode arrays [15], emphasizing the following principle: (i) neuronal activity is elicited by capacitive stimulation from a silicon chip and (ii) neuronal activity is recorded by a transistor located on the same chip. Nevertheless, the signal-tonoise ratios were relatively small often preventing the distinction between single cell recordings and multi-unit activity. Inspired by Fromherz's seminal works [16,17], Charles Lieber's team demonstrated enhanced signal-to-noise ratios using arrays of nanowire field-effect transistors connected to individual axons or dendrites of mammalian neurons [18]. In this report, each nanoscale junction was used for spatially resolving stimulations, and/or inhibitions of propagating neuronal signals with great detection sensitivity.

Moreover, neural patterning has attracted much interest in neural electronics [19] and neural engineering [20]. Compared with randomly cultured neurons, patterned neurons possess a much simpler network structure, which is easier for network modelling and signal analysis. In addition, the amplitude of detected action potentials of neurons is higher when neurons are located directly 
on the electrodes [6]. With these advantages, different protocols have been developed to perform and optimize neuron positioning and neurite growth. Thus, methods such as micro-contact printing [19], photodegradation [21], microfluidic structuration [22], biomolecule patterning [23] and photoresist processing [24] were applied for constraining neurons and guiding neurites on planar substrates.

In the present study, we have produced neural networks with defined topology on a substrate with appropriate integrated electrical contacts for each (or groups of) neuron(s). This bottom-up approach faced three objectives: i) non-invasive electrical interfacing of individual neuronal cell bodies, ii) control of neurites outgrowth to form topologically defined and stable networks, and iii) the integration of both defined networks and extracellular interfacing in a same, dedicated system. Our transistor-based biosensing interface solved these three challenges at once.

\section{Materials and Methods}

\subsection{Design and fabrication of biosensors}

Considering a $\mathrm{SiO}_{2} / \mathrm{Si}_{3} \mathrm{~N}_{4}$ pH-sensitive chemical field effect transistor ( $\mathrm{pH}-\mathrm{ChemFET}$ ) technology [25], N-channel metal-insulator-semiconductor field effect transistors (MISFET) were realized. The MISFET fabrication and encapsulation processes were previously described [26] but briefly reminded hereafter. Starting with a 6 inch, N-type $\left(1 \times 10^{13} \mathrm{~cm}^{-2}\right),(100)$ silicon wafer, Pdoped wells were realized by boron implantation $\left(1 \times 10^{15} \mathrm{~cm}^{-2}\right)$ and, following, N-doped source and drain regions were formed by arsenic implantation $\left(1 \times 10^{18} \mathrm{~cm}^{-2}\right)$. The gate was then fabricated through the stacking of thermal silicon oxide $\mathrm{SiO}_{2}$ (50 nm-thick), LPCVD silicon nitride $\mathrm{Si}_{3} \mathrm{~N}_{4}$ (50 nm-thick) and PVD titanium/gold thin films (600 nm-thick). The MISFET has a $10 \mu \mathrm{m}$-long and $200 \mu \mathrm{m}$-wide metallic gate. Wafer-level passivation was finally performed using a polymer-based technology. Figure 1 shows a schematic of the $\mathrm{SiO}_{2} / \mathrm{Si}_{3} \mathrm{~N}_{4} / \mathrm{Ti} / \mathrm{Au}$ MISFET device. A $50 \mu \mathrm{m}$-thick SU-8 photoresist layer was patterned to fabricate $10 \mu \mathrm{m}$-wide and $35 \mu \mathrm{m}$-high 3D microfluidic channels as well as micro-tanks and neurons areas of reception using the "Stepper technology" [27]. Figure 2 shows a scanning electron microscope (SEM) cross-section revealed by Secco etching [28] of a SU-8 3D micro-channel integrated on a MISFET transistor. In order to increase interactions with the future neural networks, 16 MISFET devices were realized on a same chip in conjunction with the upper SU-8 microfluidic channels (Figure 3 a). The final 16-MISFET array (1 $\mathrm{mm}^{2}$ surface) and its associated microfluidic network (Figure 3 b) are referred to as "neurochip" hereafter. Finally, the neurochips were packaged on a printed circuit board (PCB) by flip-chip 
bonding. Electronic contacts were isolated from the sensitive area thanks to an under-fill, and a Pyrex ring was glued on the PCB to create a chamber for the cultured cells [26].

\subsection{FET filter system for liquid phase measurements}

An associated electronic card was specifically developed to interface the neurochips [26]. Thanks to this set-up, it is possible to multiplex the 16 MISFET on the neurochip while filtering any noise coming from the electronic interface and/or the liquid. All devices were biased using the same electrical configuration: a silver / silver chloride $(\mathrm{Ag} / \mathrm{AgCl})$ wire was used to apply the gate voltage to the neuronal culture medium and the same drain source current and voltage (IDS $=250 \mu \mathrm{A}$ and $\mathrm{V}_{\mathrm{DS}}=2 \mathrm{~V}$ ). Thus, the different measurements related to neuronal action potentials were comparable from one MISFET to the other. The resting potential was fixed to $0 \mathrm{~V}$ due to the removal of any direct current (DC) component.

\subsection{Rat retina neuronal cell culture}

Cell cultures were prepared from Long-Evans rats, which were purchased from Janvier Company (Le Genest Saint-Isle, France). All experiments were carried out in accordance with European Community Council Directives (86/609/EEC) and with the ARVO (Association for Research in Vision and Ophthalmology) statement for the use of animals in ophthalmic and visual research. Ethics committee approval is not required for cell culture experiments, as stated in European Union Directives (2010/63/UE). Animals were killed by $\mathrm{CO}_{2}$ sedation and cervical elongation, and all efforts were made to minimize suffering.

Neuronal cells were isolated from the retina of newborn Long-Evans rats (postnatal day 7), with an immunopanning technique, according to protocols previously described in young rats [29]. The chips were coated with poly-D-lysine ( $2 \mu \mathrm{g} / \mathrm{cm}^{2}$ for $45 \mathrm{~min}$; Sigma-Aldrich) followed by laminin (1 $\mu \mathrm{g} / \mathrm{cm}^{2}$ overnight; Sigma-Aldrich). Cells were seeded in ND-G medium at an initial density of $4 \mathrm{x}$ $10^{4}$ cells $/ \mathrm{cm}^{2}$ on SU-8 microfluidic samples. The cultures were kept in an incubator at $37^{\circ} \mathrm{C}$ with $5 \% \mathrm{CO}_{2}$ for three days. Then, after this three-day period, optical characterisations of neurons growth were immediately performed at room temperature.

\subsection{Lymnaea Stagnalis neuronal cells culture}

Lymnaea Stagnalis (freshwater snails) were purchased from the National Institute of Agronomic Researches (INRA) of Rennes (France), namely from the Experimental Unit of Aquatic Ecology 
and Ecotoxicology section. Laboratory-raised stocks of Lymnaea Stagnalis were maintained in aquaria filled with well-aerated filtered water at $22^{\circ} \mathrm{C}$. Experiments were performed on 3 month-old snails (20-30 millimeters in length). Snail neurons were chosen since, first, they exhibit high action potentials (10-100 mV) and, second, their culture and electrophysiological characterization are performed at room temperature, i.e. with no need for temperature control [30-34]. Thus, their isolation followed previously established protocols [30-32]. Lymnaea Stagnalis were de-shelled, soaked in $25 \%$ lysterin in normal saline consisting of $51.3 \mathrm{mM} \mathrm{NaCl}, 1.7 \mathrm{mM} \mathrm{KCl}, 4.1 \mathrm{mM} \mathrm{CaCl}_{2}$, $1.5 \mathrm{mM} \mathrm{MgCl}_{2}$ and $5.0 \mathrm{mM}$ HEPES at $\mathrm{pH} 7.9$ (all from Sigma-Aldrich) for 10 minutes. Central ganglionic rings were isolated from Lymnaea in normal saline and put in $150 \mu \mathrm{g} / \mathrm{mL}$ gentamicin for 10 minutes at room temperature. Left and right pedal ganglia were isolated from the rest of the brain and treated with $1.33 \mathrm{mg} / \mathrm{mL}$ collagenase and $0.67 \mathrm{mg} / \mathrm{mL}$ trypsin (all Sigma-Aldrich) for 30 minutes. Pedal ganglia were washed several times in Leibovitz L15 medium (Sigma-Aldrich). Ganglia were dislocated using a micropipette in Leibovitz L15 medium while sucking, and the contents were dispensed back into the same container. The operation was repeated until most tissues were dispersed. Neuronal solution was divided up on different chips previously coated with poly-Dlysine $\left(2 \mu \mathrm{g} / \mathrm{cm}^{2}\right.$ for 45 minutes; Sigma-Aldrich) followed by laminin $\left(1 \mu \mathrm{g} / \mathrm{cm}^{2}\right.$ overnight; SigmaAldrich). The completed medium consisting of Leibovitz L15, $20 \mu \mathrm{g} / \mathrm{mL}$ gentamycin, $30 \mathrm{mM}$ glucose and $10 \mathrm{ng} / \mathrm{mL}$ nerve growth factor (NGF), was applied to different chambers (250 $\mu \mathrm{L} /$ chambers). The neurons were incubated for three days at room temperature (around $21^{\circ} \mathrm{C}$ ). Finally, all optical characterizations and electrical measurements with snails' pedal neurons were performed at room temperature.

\subsection{Cell viability assessment}

Neuronal cells were visualized with the "lived-dead" test (Life Technologies) that consists in labelling viable cells with calcein-AM being detected as green fluorescence, whereas dead cells were labelled with ethidium iodide producing a red fluorescence. Briefly, after three days in culture, chips bearing SU-8 3D microfluidics were incubated in a mixture of calcein-AM and ethidium homodimer-1 (diluted in a phosphate buffer medium PBS, $\mathrm{pH}=7.4$ ) for one hour in a humidified chamber $\left(37^{\circ} \mathrm{C}, 5 \% \mathrm{CO}_{2}\right)$. Calcein-AM-positive viable cells were visualized under an epifluorescence microscope (Leica DM 5000B, Solms, Germany). The silicon chips were mounted with Fluorsave reagent (Calbiochem, San Diego, CA, USA) and viewed with an upright confocal microscope (Nikon, Tokyo, Japan). The chips emitted a blue self-fluorescence (excitation at 408 $\mathrm{nm}$ ) and the cells a green fluorescence (excitation at $488 \mathrm{~nm}$ ). 


\subsection{Neuronal cell fixation for electron microscopy observations}

After checking the viability of cells on each chip using epifluorescence microscopy, the culture medium was removed. For fixation, the chips were rinsed with phosphate buffered saline (PBS) and then incubated in $2.5 \%$ glutaraldehyde solution (Sigma-Aldrich) for 12 hours at $4^{\circ} \mathrm{C}$. Then, the glutaraldehyde solution was removed and chips were rinsed with PBS. For cell dehydration, we used different ethanol solutions with increasing concentrations in water. The cells were therefore incubated in 50\%, 70\%, $90 \%$ and $100 \%$ of ethanol for 10 minutes each. After the last dehydration step, the fixated samples were air dried for a day to remove all alcoholic residues before observation under a scanning electron microscope (SEM).

\section{7. $\quad$ Toxin treatments for modulating action potentials activities}

Electrical measurements of neuronal activities were only performed on Lymnaea Stagnalis pedal neurons at room temperature as previously established in the literature [30-34].

For the action potential recordings, two different toxins were used to modulate neuronal activity. The first was picrotoxin, which is known to excite silent cells [35]. We used $100 \mu \mathrm{M}$ of picrotoxin to trigger neuronal activity. Gamma-amynobutyric acid (GABA), an antagonist of picrotoxin, was also tested in order to inhibit electrical firing of action potentials: $100 \mu \mathrm{M}$ of GABA was necessary to reverse picrotoxin-induced excitation. As a result, picrotoxin - GABA - picrotoxin sequential injections were performed in order to reverse the action induced by the other, thus modulating neuronal activity.

Finally, a cleaning procedure was developed for our MISFET chip. Medium with toxin was removed thanks to a rinse cycle with deionised water. Then, the SU-8 microfluidic system was filled with classic liquid hand soap, cleaned with a soft mechanical cleaning technique, and rinsed finally with water. After undergoing the preliminary treatments as described before cells could again be cultured and the neurochip could be reused. We were able to use the biosensor for more than 15 cultures without degrading the SU-8 micro channels and/or the MISFET structures.

\section{Results and discussion}

\subsection{Neuronal orientation}

The first objective of our work was to generate on-chip guided growth of the neurons in order to increase their contact with our MISFET sensors. We first evaluated if SU-8 micro-channels above 
the transistors' gates (Figure 3) could induce the neuronal guided growth. To overcome the wellknown SU-8 cytotoxicity [36], we proceeded to a four-step specific protocol necessary to turn the SU-8 biocompatible. The first step is chemical treatments with SU-8 developer in order to remove all resist residues thanks to SU-8 over-development. The second step is a photolithographic one that allows to reflow the SU-8 resist across all its thickness thanks to a one minute UV exposure at a 365 nm wavelength. The third step is a thermal annealing at $150^{\circ} \mathrm{C}$ during three days to eliminate all solvents still trapped inside the SU-8 layer. The final step is an $\mathrm{O}_{2}$ plasma treatment to render the SU-8 surface hydrophilic [37]. SEM observations and measures before and after the specific treatment have shown that this protocol do not affect the final dimension of SU-8 structures or adhesion between the SU-8 layer and the substrate nor does it induce cracks in the SU-8 layer. After performing the protocol, we deposited poly-D-lysine and coated using laminin in order to create a favourable substrate that improves the efficiency of cell expansion and neuronal differentiation [38].

Rat retina neurons were cultured on the neurochips previously coated with poly-D-lysine and laminin. As mentioned previously (cf. 2.3), rat retina neurons were kept in a humidified chamber $\left(37^{\circ} \mathrm{C}, 5 \% \mathrm{CO}_{2}\right)$ for three days. Neuronal growth was assessed by microscopy and cell viability was tested thanks to viability test described previously. An epifluorescence microscope (Leica DM4000 B LED) was used to visualize viable cells showing that more than $95 \%$ of cells survived on the chip after three days of culture at $37^{\circ} \mathrm{C}$. Rat retinal neurons limited their development to the reception areas and towards the SU-8 micro channels. These first results demonstrate the guided neurites growth towards the MISFET gate. Neurons were then examined at a smaller resolution to define if they constitute a neuronal network on the neurochip. Confocal and fluorescence spectroscopies were used to visualize neurons on the surface for two different microchannels configurations (Figures 4 and 5). Figures 4, 5 and 6 illustrate that many neurons can be seen on the reception areas according to their size, and their neurites appeared to be guided across the different micro channels thanks to the SU-8 layer microstructures thereby leading to the creation of a neuronal network. After the culture period, we used a protocol described above (cf. 2.5 and 2.6) to fix and dehydrate neurons for SEM observations. Figure 6 illustrates neurons with neurites in the SU-8 microchannels and the absence of neurites growth on the micro-channels walls towards the SU-8 upper layer (height: $50 \mu \mathrm{m}$ ). Rat retina neuronal recording was tested using our FET filter system. However, these specific neurons produce low voltage $(\sim 100 \mu \mathrm{V})$ [39] that could not be recorded by our electronic interface. Indeed, even using an adapted electronic feedback control to reduce the electrochemical noise [26], values around $500 \mu \mathrm{V}$ were still obtained. In consequence, we could not record neuronal activity from rat retina neurons. This is why we used snails' pedal neurons that produce stronger signals. 


\subsection{Action potential recording from Lymnaea Stagnalis pedal neurons}

Since the action potential of rat retina neurons was not compatible with our measurement noise, the second aim of this work, namely to measure neuronal activity, was achieved with the use of Lymnaea Stagnalis snail pedal neurons that are associated with higher voltage $(>10 \mathrm{mV})$. The neurochips were functionalized as previously described and snail neuronal cells were cultured for three days at room temperature (around $21^{\circ} \mathrm{C}$ ) according to the previously described protocol (cf. 2.4). Viability test (cf. 2.5.) was realized to check the biocompatibility between snail neurons and neurochip devices (Figure 7). As for mammalian neurons, snail neurons were deposited on the biosensor and survived easily with more than a $90 \%$ survival rate. After the cell fixation procedure (cf. 2.6.), SEM observations revealed that the reception areas were well suited for this kind of neuron cells since there was usually only a single cell by each electrode/gate (Figure 8). As a result, measurement of a single cell was expected, even if, in some case, a second cell was also fixed on a given MISFET gate. Neurites were again oriented by the SU-8 microstructures.

After the successful cell culture and guided growth, electrical measurements were made. An output voltage of $0 \mathrm{~V}$ was measured in the resting state of the neurons and, except for the measurement noise $(\sim 500 \mu \mathrm{V})$, no electrical signals were found without any neurons culture. However, as soon as a neural network was grown on the neurochip, some occasional events were measured with the difference between the maximal and the resting potential around $100 \mathrm{mV}$ (figure 9). In $80 \%$ of our measurements, randomly triggered spiked-like events were recorded. In these cases, the duration between the start of the depolarisation and the hyperpolarisation endpoint being estimated to be around $7 \mathrm{~ms}$. In the other cases, periodic electrical phenomena were evidenced. According to literature [30-32,40], we assumed to have detected action potentials. Nevertheless, to demonstrate that they events were truly neuronal spikes, we decided to use a "picrotoxin - GABA picrotoxin" protocol.

First, we applied picrotoxin in the bath to increase the firing frequency and the quality of the recording signal. Figure 10 illustrates the change in spike-like events after applying picrotoxin. Under the influence of the picrotoxin, the waveform of the spike-like events were greatly modified as both the signal amplitude and its duration decreased to $30 \mathrm{mV}$ and $0.5 \mathrm{~ms}$ respectively (Figure 11 $\mathrm{a}$ and $\mathrm{b}$ ). The change observed after adding $100 \mu \mathrm{M}$ of picrotoxin in the perfusion medium was not seen during the first 30 seconds as expected since the picrotoxin must diffuse and activate the neuronal $\mathrm{GABA}_{\mathrm{A}}$ receptors. After this delay of 30 seconds, action potentials were measured on the MISFET channels and their frequency greatly increased one minute later. As a consequence, neurons exhibited bursts of spike-like events with amplitude reaching $30 \mathrm{mV}$ and a period around 
$0.5 \mathrm{~ms}$ (Figure 11). This time is defined as the so-called "spiking frequency" and is around $2000 \mathrm{~Hz}$. Picrotoxin was therefore responsible for an increase in the spiking frequency from $1 \mathrm{~Hz}$ (minimal measurement value) to $2000 \mathrm{~Hz}$. Five minutes after the picrotoxin injection, the analysed medium was replaced by a new medium containing this time $100 \mu \mathrm{M}$ of GABA. As for picrotoxin, GABA diffusion required some time to activate $\mathrm{GABA}_{\mathrm{A}}$ receptors and to inhibit the neurons. After 30 seconds, the triggering frequency decreased progressively taking more than two minutes to washout the picrotoxin effect returning neurons to their initial resting potential $(0 \mathrm{~V})$. Five minutes later, the perfused medium was changed back to the picrotoxin containing solution. Again, neurons generated some bursting events after one minute. The changes in the recorded signals when exchanging the GABA inhibition with picrotoxin indicated that the MISFET biosensor measures effectively neuronal activities.

\section{Conclusion}

In this study, we developed a MISFET transistor array to record neuronal activities while using integrated SU-8 microfluidic structures in order to guide the neuronal growth. This lab-on-chip was tested for the organized growth of rat retina neurons and Lymnaea Stagnalis snail pedal neurons. Then, the MISFET array was used for the recording of action potentials while using Lymnaea Stagnalis pedal neurons. Neuronal activities were successfully triggered and inhibited by implementing a picrotoxin - GABA - picrotoxin successive injections protocol. The recorded signals are consistent with recorded neuronal activities for snail neurons in culture demonstrating thereby that our MISFET transistors can be used to measure neuronal activities.

Future works will concern the improvement of our "neurochip" platform. First of all, the measurement noise has to be decreased drastically $(<50 \mu \mathrm{V})$ in order to be compatible with the recording of mammals neurons and to be finally able to monitor action potentials from rat retina neurons. This will be performed with the development of specific low-noise electronic interface, either by improving the electronic feedback control to reduce the electrochemical noise, either by increasing the acquisition data speed and using high-frequency filter to reduce the electronic noise. So, the 16-MISFET neurochip will be used to analyse neural networks, aiming to the global monitoring of electrophysiological signals. Secondly, the MISFET devices have to be replaced by ISFET (Ion-Sensitive Field Effect transistor) devices in order to be able to monitor ionic concentrations variations (focusing especially on the sodium $\mathrm{Na}^{+}$and potassium $\mathrm{K}^{+}$ions) on the neuron surface and to correlate them with the neuronal activities. Finally, the "Stepper technology" has still to be studied in order to optimize the mass fabrication of SU-8 3D micro-templates for the neuronal growth. 


\section{Acknowledgments}

Amel Bendali received a PhD fellowship from the University "Pierre and Marie Curie" (Paris 06). All authors were supported by a CNRS grant (programme interdisciplinaire: interface physique chimie). Serge Picaud was supported by grants from the French "Fondation pour la Recherche Médicale" (FRM) and by the European Community's Seventh Framework Programme (FP7) under grant agreement $\mathrm{n}^{\circ} 280433$ (NEUROCARE project). The technological realizations and associated research works were partly supported by the French RENATECH network.

\section{References}

[1] F.A. Edwards, A. Konnerth, B. Sakmann, T. Takahashi, "A thin slice preparation for patch clamp recordings from neurones of the mammalian central nervous system", European Journal of Physiology, 414 (1989) 600-612.

[2] O. Marre, A. Dario, N. Deshmukh, K. Sadeghi, F. Soo, T.E. Holy, M.J. Berry II, " Mapping a complete neural population in the retina", Journal of Neurosciences, 32 (2012) 14859-73.

[3] M.E. Spira, A. Hai, "Multi-electrode array technologies for neuroscience and cardiology", Nature Nanotechnology, 8 (2013) 83-94.

[4] F.O. Morin, Y. Takamura, E. Tamiya, "Investigating neuronal activity with planar microelectrode arrays: achievements and new perspectives", Journal of Bioscience and Bioengineering, 100 (2005) 131-143.

[5] C. Xie, Z. Lin, L. Hanson, Y. Cui, B. Cui B., "Intracellular recording of action potentials by naopillar electroporation", Nature Nanotechnology 7 (2012) 185-190.

[6] Y. Nam, J. Chang, D. Khatami, G.J. Brewer, B.C. Wheeler, " Gold-coated microelectrode array with thiol linked self-assembled monolayers for engineering neuronal cultures ", IEEE Proceedings Nanobiotechnology, 151 (2004) 109-115.

[7] D. Ghezzi, M.R. Antognazza, M. Dal Maschio, E. Lanzarini, F. Benfenati, G. Lanzani, " A hybrid bioorganic interface for neuronal photoactivation ", Nature Communications, 166 (2011) $1-7$.

[8] G. Buzsáki, C.A. Anastassiou, C. Koch, "The origin of extracellular field and currents - EEG, ECoG, LFP and spikes", Neuroscience, 13 (2012) 407-420.

[9] T. Cramer, B. Chelli, M. Murgia, M. Barbalinardo, E. Bystrenova, D.M. De Leeuw, F. Biscarini, "Organic ultra-thin film transistors with a liquid gate for extracellular stimulation and 
recording of electric activity of stem cell-derived neuronal networks", Physical Chemistry Chemical Physics, 15 (2013) 3897-3905.

[10] L. Berdondini, K. Imfeld, A. Maccione, M. Tedesco, S. Neukom, M. Koudelka-Hep, S. Martinoia, "Active pixel sensor array for high spatio-temporal resolution electrophysiological recordings from single cell to large scale neuronal networks", Lab on a chip, 9 (2009) 26442651.

[11] A. Hierlemann, U. Frey, S. Hafizovic, F. Heer, "Growing cells atop microelectronic chips: Interfacing electrogenic cells in vitro with CMOS-based microelectrode arrays", Proceedings of the IEEE, 99 (2011) 252-284.

[12] S. Herwik, S. Kisban, A. Aarts, K. Seidl, G. Girardeau, K. Benchenane, M.B. Zugaro, S.I. Wiener, O. Paul, H.P. Neves, P. Ruther, "Fabrication technology for silicon-based microprobe arrays used in acute and sub-chronic neural recording", Journal of Micromechanics and Microengineering, 19 (2009) 1-11.

[13] J. Pine, "Recording action potentials from cultured neurons with extracellular microcircuit electrodes", Journal of Neuroscience Methods, 2 (1980) 19-31.

[14] G.W. Gross, A.N. Williams, J.H. Luca, "Recording of spontaneous activity with photoetched microelectrode surfaces from mouse signal neurons in culture", Journal of Neuroscience Methods, 5 (1982) 13-22.

[15] P. Fromherz, "Electrical interfacing of nerve cells and semiconductor chips", ChemPhysChem, 3 (2002) 276-284.

[16] P. Fromherz, O. Müller, R. Weis, "Neuron transistor: electrical transfer function measured by the patch-clamp technique", Physical Review Letters, 71 (1993) 4079-4082.

[17] P. Fromherz, A. Stett, "Silicon-neuron junction: capacitive stimulation of an individual neuron on a silicon chip", Physical Review Letters, 75 (1995) 1670-1673.

[18] F. Patolsky, B. Timko, G. Yu, Y. Fang, A. Greytak, G. Zheng, C.M. Lieber, "Detection, stimulation, and inhibition of neuronal signals with high-density nanowire transistor arrays", Science, 313 (2006) 1100-1104.

[19] M. Jungblut, W. Knoll, C. Thielemann, M. Pottek, "Triangular neuronal network on microelectrode arrays: an approach to improve the properties of low-density networks for extracellular recording", Biomedical Microdevices, 11 (2009) 1269-1278.

[20] M.R. Hynd, J.P. Frampton, N. Dowell-Mesfin, J.N. Turner, W. Shain, "Directed cell growth on protein-functionalized hydrogel surfaces", Journal of Neuroscience Methods, 162 (2007) 255263. 
[21] N.S. Baek, J.-H. Lee, Y.H. Kim, B.J. Lee, G.H. Kim, I.-H. Kim, M.-A. Chung, S.-D. Jung, "Photopatterning of cell-adhesive-modified poly(ethyleneimine) for guided neuronal growth", Langmuir 27 (2011) 2717-2722.

[22] J. Wang, L. Ren, L. Li, W. Liu, J. Zhou, W. Yu, D. Tong, S. Chen, "Microfluidics: a new cosset for neurobiology", Lab on a chip, 9 (2009) 644-652.

[23] J. Cheng, G. Zhu, L. Wu, X. Du, H. Zhang, B. Wolfrum, Q. Jin, J. Zhao, A. Offenhäusser, Y. $\mathrm{Xu}$, "Photopatterning of self-assembled poly(ethylene)glycol monolayer for neuronal network fabrication", Journal of Neuroscience Methods, 213 (2013) 196-203.

[24] W.C. Chang, D.W. Sretavan, "Novel high-resolution micropatterning for neuron culture using polylysine adsorption on a cell repellant, plasma-polymerized background", Langmuir 24 (2008) 13048-13057.

[25] I. Humenyuk, B. Torbiéro, S. Assié Souleille, R. Colin, X. Dollat, B. Franc, A. Martinez, P. Temple-Boyer, "Development of $\mathrm{pNH}_{4}$-ISFET microsensors for water analysis", Microelectronics Journal, 37 (2006) 475-479.

[26] F. Larramendy, F. Mathieu, S. Charlot, L. Nicu, P. Temple-Boyer, "Parallel detection in liquid phase of N-channel MOSFET/ChemFET microdevices using saturation mode", Sensors and Actuators B, 176 (2013) 379-385.

[27] F. Larramendy, L. Mazenq, P. Temple-Boyer, L. Nicu, "Three-dimensional close microfluidic channels fabrication by stepper projection single step lithography: the diabolo effect", Lab on a chip, 12 (2012) 387-390.

[28] J. Secco d'Aragona, "Dislocation etch for (100) planes in silicon", Journal of the electrochemical society, 119 (1972) 948-952.

[29] B.A. Barres, B.E. Silverstein, D.P. Corey, L.L.Y. Chun, "Immunological, morphological, and electrophysiological variation among retinal ganglion cells purified by panning", Neuron, 1 (1988) 791-803.

[30] M. Kyriakides, C.R. Mac Crhan, C.T. Slade, N.I. Syed, W. Winlow: "The morphology and electrophysiology of the neurones of the paired pedal ganglia of Lymnae Stagnalis", Comparative Biochemistry and Physiology 93 (1989) 861-876.

[31] R.L. Ridgway, N.I. Syed, K. Lukowiak, A.G.M. Bulloch, " Nerve growth factor (NGF) induces sprouting of specific neurons of the snail Lymnaea stagnalis", Journal of Neurobiology, 22 (1991) 377-390.

[32] A.A. Prinz, P. Fromherz, "Electrical synapses by guided growth of cultured neurons from the snail Lymnaea stagnalis", Biological Cybernetics, 82 (2000) L1-L5.

[33] M. Jenkner, B. Müller, P. Fromherz, "Interfacing a silicon chip to pairs of snail neurons connected by electrical synapses", Biological Cybernetics, 84 (2001) 239-249. 
[34] A. Cohen, M.E. Spira, S. Yitshaik, G. Borghs, O. Schwartzglass, J. Shappir, "Depletion type floating gate p-channel MOS transistors for recording action potentials generated by cultured neurons", Biosensors and Bioelectronics, 19 (2004) 1703-1709.

[35] F. Moccia, C. Di Cristo, W. Winlow, A. Di Cosmo, "GABAA-and AMPA-like receptors modulate the activity of an identified neuron within the central pattern generator of the pond snail Lymnaea stagnalis", Invertebrate Neuroscience, 9 (2009) 29-41.

[36] V.N. Vernekar, D.K. Cullen, N. Fogleman, Y. Choi, A.J. Garcia, M.G. Allen, G.J. Brewer, M.C. La Placa, "SU-8 2000 rendered cytocompatible for neuronal bioMEMS applications", Journal of Biomedical Materials Research A, 89 (2008) 138-151.

[37] F. Walther, P. Davydovskaya, S. Zürcher, M. Kaiser, H. Herberg, A.M. Gigler, R.W. Stark, "Stability of the hydrophilic behavior of oxygen plasma activated SU-8", Journal of Micromechanical Microengineering, 17 (2007) 524-531.

[38] L. Qiana, W.M. Saltzman, "Improving the expansion and neuronal differentiation of mesenchymal stem cells through culture surface modification", Biomaterials, 25 (2004) 13311337.

[39] B. Kolomiets, E. Dubus, M. Simonutti, S. Rosolen, J.A. Sahel, S. Picaud, "Late histological and functional changes in the $\mathrm{P} 23 \mathrm{H}$ rat retina after photoreceptor loss", Neurobiology of Disease, 38 (2010) 47-58.

[40] G.E. Spencer, N.I. Syed, K. Lukowiak, "Neural changes after operant conditioning of the aerial respiratory behaviour in Lymnae Stagnalis", The journal of neuroscience, 19 (1999) 1835-1843.

\section{Biographies}

Florian Larramendy was born in Brive-la-Gaillarde, France, in 1986. He received the M.S. degree in Microelectronic System Engineering from the Paul Sabatier University, Toulouse, France, in 2009. He joined the Laboratory for Analysis and Architecture of Systems (LAAS), National Center of Scientific Research (CNRS), Toulouse in 2009 as PhD Student. He was in charge of the development of microsystems to measure neuronal activities. After obtaining his D. Sc. Degree in 2013, he joined the EUJO-LIMMS project in the Microsystem Materials Laboratory, Department of Microsystems Engineering (IMTEK), Freiburg, Germany, and the Laboratory for Integrated Micro Mechatronic Systems (LIMMS), Tokyo, Japan. Currently, he is in charge of the development of microprobes for brain insertion for biomedical applications. 
Amel Bendali received her BS degree in Physics in 2006 and her MS degree in Instrumentation for Biotechnology in 2008 from Institut National Polytechnique de Grenoble, France. She recently graduated her PhD in Neuroscience at Université Paris VI under the supervision of Dr Serge Picaud. Her research focuses on building networks of retinal neurons on multi-electrode arrays for the study of retinal signal processing and testing carbon-based materials such as diamond and graphene to design retinal prosthetic devices.

Marie Charline Blatché was born in Toulouse, France, in 1986. She received the Licence degree in Biotechnologies from Nice-Sophia Antipolis University, Nice, France in 2008. She joined the Laboratoire d'Analyse et d'Architecture des Systèmes (LAAS), Centre National de la Recherche Scientifique (CNRS), Toulouse in 2009, where she is in charge of the "Biology" technological platform and worked on the development of cell growth on microsystems.

Fabrice Mathieu was born in Orléans, France, in 1972. He received the engineering degree in communication systems and electronics from the CNAM (Conservatoire National des Arts et Métiers), Toulouse, France, in 2003. He joined the Laboratoire d'Analyse et d'Architecture des Systèmes (LAAS), Centre National de la Recherche Scientifique (CNRS), Toulouse, in 2001, where he is currently in charge of the development and design of very low signal detection systems applied to the micro(nano)electromechanical systems area and its complete electronic treatment and control for automation.

Serge Picaud was born in Paris (France) in 1961. He received a $\mathrm{PhD}$ in Neurosciences from the University of Marseille in 1990. He was educated by leaders in the field of retinal physiology during doctoral and postdoctoral stays with Professor Wässle (Max-Planck Institute for Brain research, Francfurt, Germany) and Prpfessor Werblin (University of California, Berkeley, USA). He is currently leading the team "retinal information processing" at the Vision Institute in Paris created by Professor Sahel. After studying retinal physiology and pharmacology with applications in neuroprotection, the team has moved toward strategies for restoring vision in blind patients having lost their photoreceptors. These strategies involve retinal prostheses or optogenetic therapy, which requires expression of photosensitive pump or ionic channels in selected retinal neurones.

Pierre Temple-Boyer was born in Montpellier (France) in 1966. He received his Engineer Master's Degree in electronic engineering from the Ecole Supérieure d'Electricite (Paris - France) in 1990 and his Master's Degree in microelectronics from the Université Paul Sabatier de Toulouse (France) in 1992. He joined the Laboratoire d'Architecture et d'Analyse des Systèmes of the French 
Centre National de la Recherche Scientifique (LAAS-CNRS) in 1992 and received the PhD degree from the Institut National des Sciences Appliquées de Toulouse (France) in 1995. Since then, as a senior researcher, he has been working on the development of micro- and nanotechnologies.

Liviu Nicu was born in 1973 in Bucharest (Romania). After completing his Master of Electrical Engineering at the Paul Sabatier University of Toulouse (France) in 1997, he joined the Integrated Microsystems Group at the LAAS (Laboratory for Analysis and Architecture of Systems) of Toulouse where he obtained his Ph.D. in 2000 into the Micromechanical Structures field. From 2000 to 2003, he was R\&D Engineer at Thales Avionics, Valence (France). His activities focused onto the development of micromechanical sensors for the civil and military navigation applications. Since 2003 he joined the NanoBioSystems Group at LAAS as a full time CNRS (National Center of Scientific Research) researcher where he currently works in two main research fields: the development of (1) new resonant bio(chemical)sensors using M(N)EMS technologies and of (2) cantilever-based microsystems for contact deposition of small amounts of biological samples for biochip applications. 


\section{FIGURE CAPTIONS}

Fig. 1. Schematic of the $\mathrm{SiO}_{2} / \mathrm{Si}_{3} \mathrm{~N}_{4} / \mathrm{Ti} / \mathrm{Au}$ MISFET device

Fig. 2. Picture of SU-8 3D micro-channel fabricated using the "Stepper technology" on a MISFET

Fig. 3. Optical microscopy photographs of a) (left) the 16 MISFET array and its associated SU-8 3D microfluidic network and b) (right) the neurochip bearing the SU-8 microfluidics

Fig. 4. Confocal microscopy photograph of neurons network developed through SU-8 3D microfluidic structures

Fig. 5. Fluorescence picture of calcein-stained viable neurons and their outgrowths in culture on MISFETs and across SU-8 3D micro channels

Fig. 6. SEM picture of neurons having developed neurites through SU-8 3D micro channel

Fig. 7. Fluorescence observed of a) live cells and b) dead cells on biosensor

Fig. 8. SEM picture of two snail neurons fixed on a MISFET gate

Fig. 9. Random triggered action potential recording

Fig. 10. The recorded extracellular potentials to $100 \mu \mathrm{M}$ of picrotoxin stimuli

Fig. 11. "Spike type" complete waveform of recorded neuron potentials

a) without and b) with $100 \mu \mathrm{M}$ of picrotoxin 


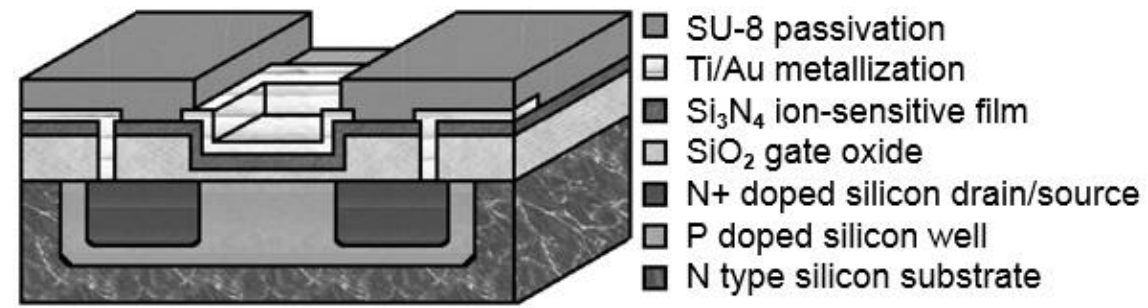

Fig. 1. Schematic of the $\mathrm{SiO}_{2} / \mathrm{Si}_{3} \mathrm{~N}_{4} / \mathrm{Ti} / \mathrm{Au}$ MISFET device 


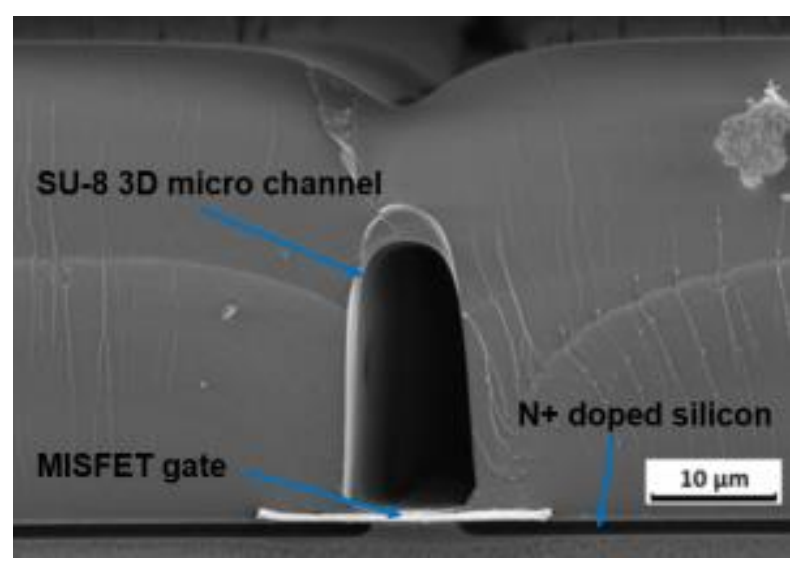

Fig. 2. Picture of SU-8 3D micro-channel fabricated using the "Stepper technology" on a MISFET 

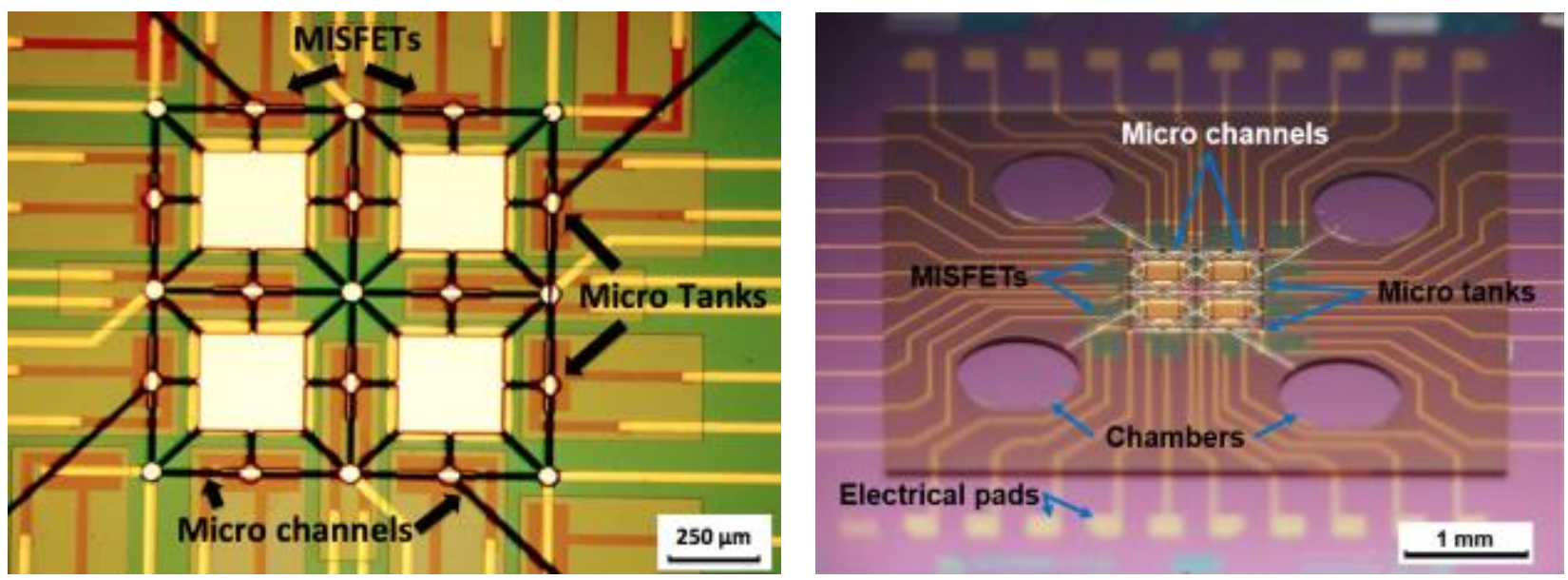

Fig. 3. Optical microscopy photographs of a) (left) the 16 MISFET array and its associated SU-8 3D microfluidic network and b) (right) the neurochip bearing the SU-8 microfluidics 


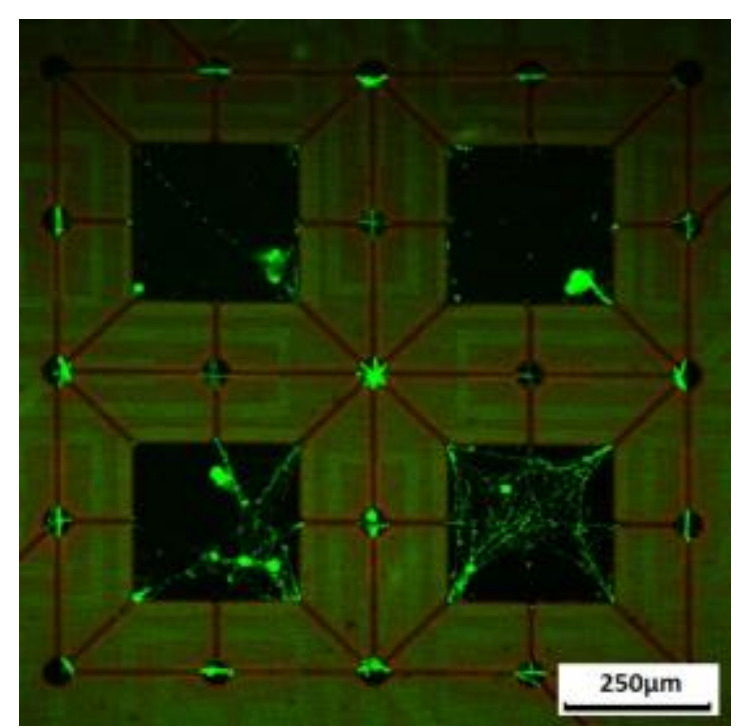

Fig. 4. Confocal microscopy photograph of neurons network developed through SU-8 3D microfluidic structures 


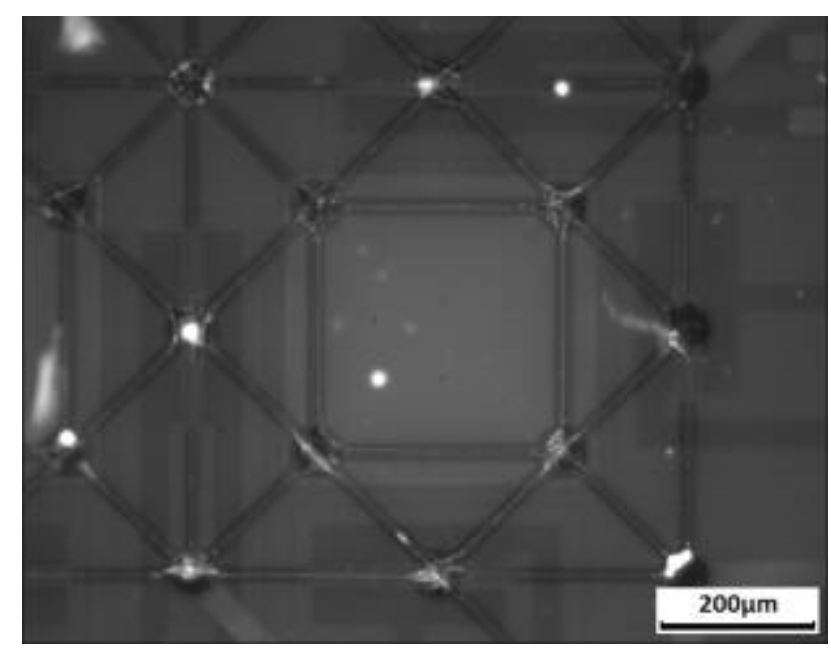

Fig. 5. Fluorescence picture of calcein-stained viable neurons and their outgrowths in culture on MISFETs and across SU-8 3D micro channels 


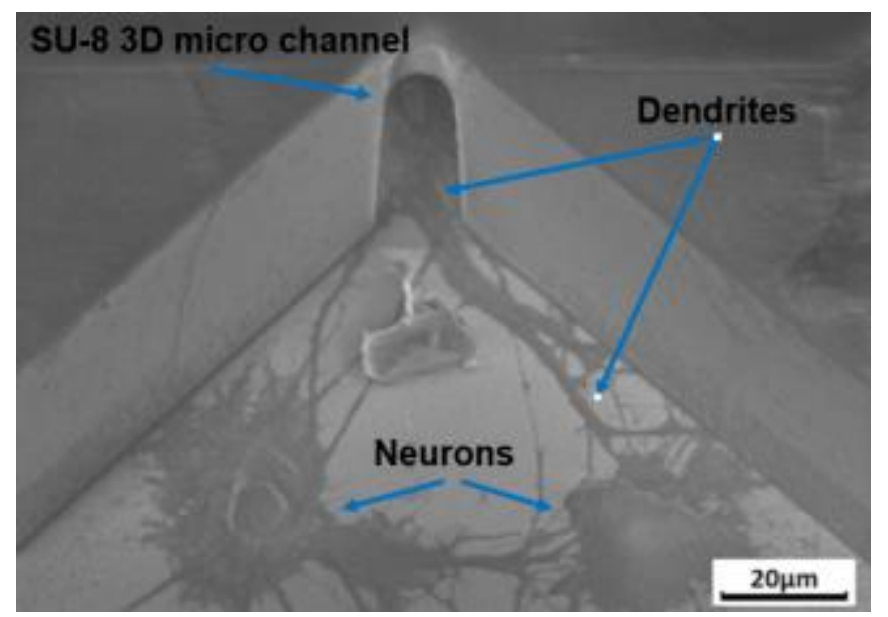

Fig. 6. SEM picture of neurons having developed neurites through SU-8 3D micro channel 

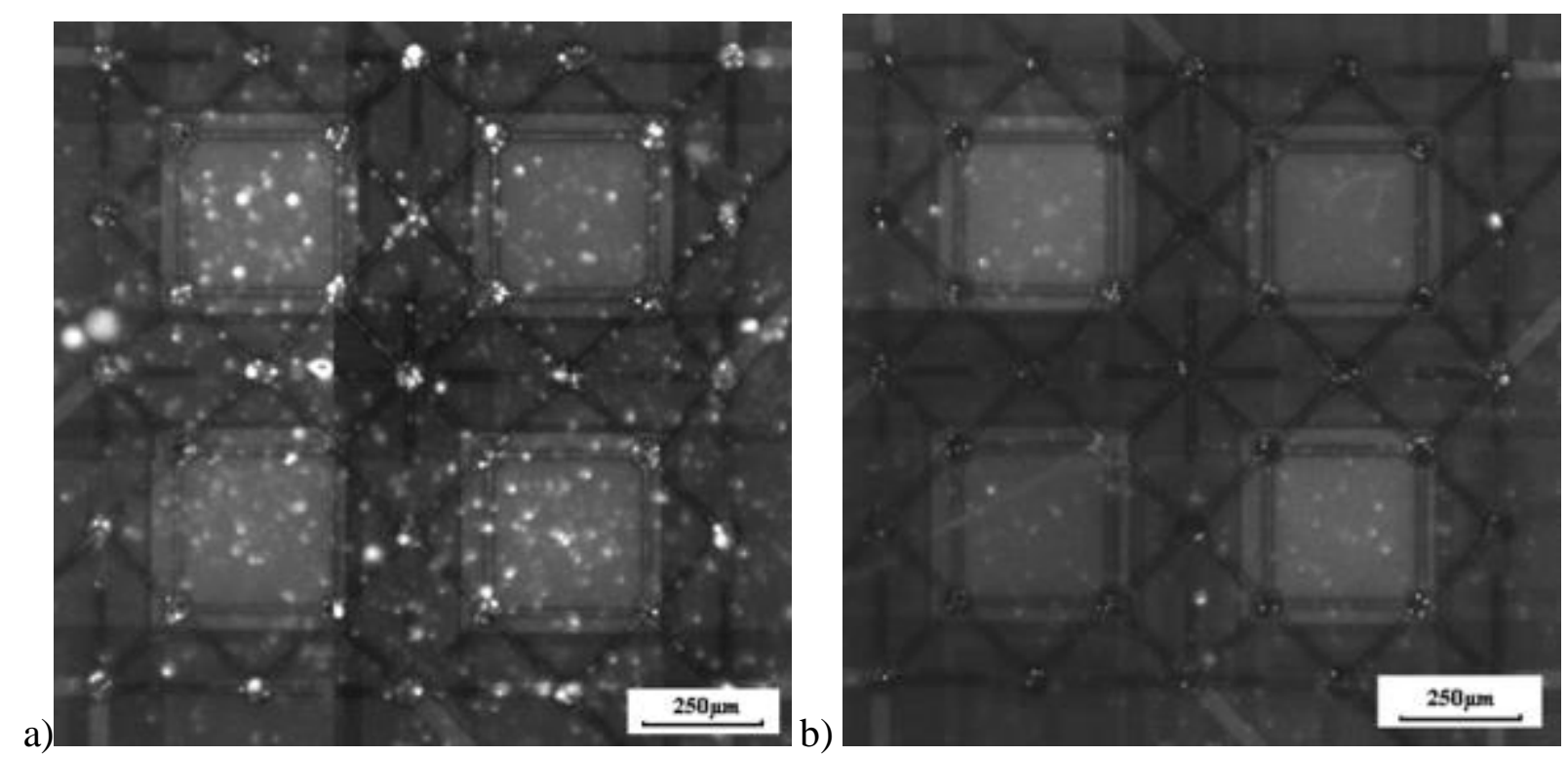

Fig. 7. Fluorescence observed of a) live cells and b) dead cells on biosensor 


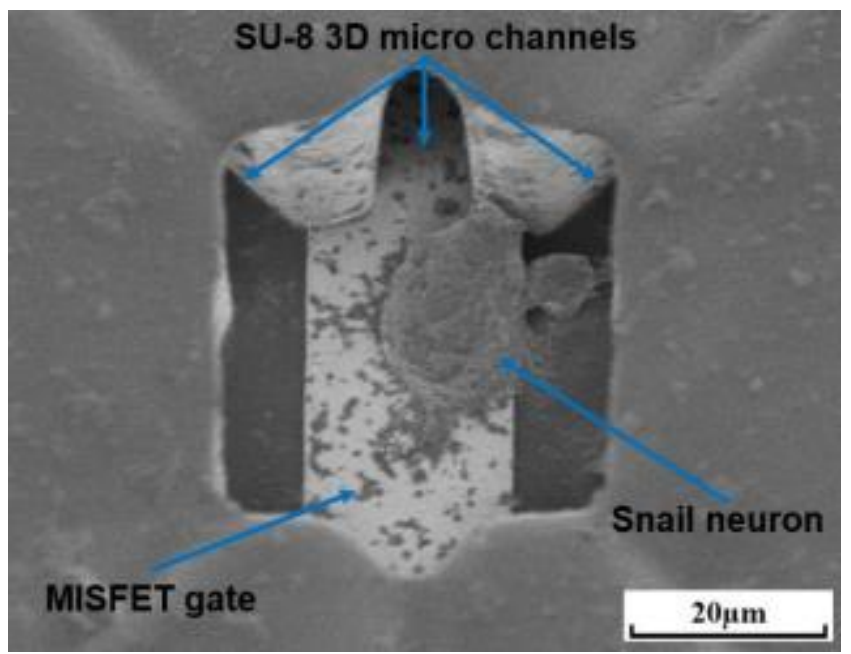

Fig. 8. SEM picture of snail neuron fixed on a MISFET gate 


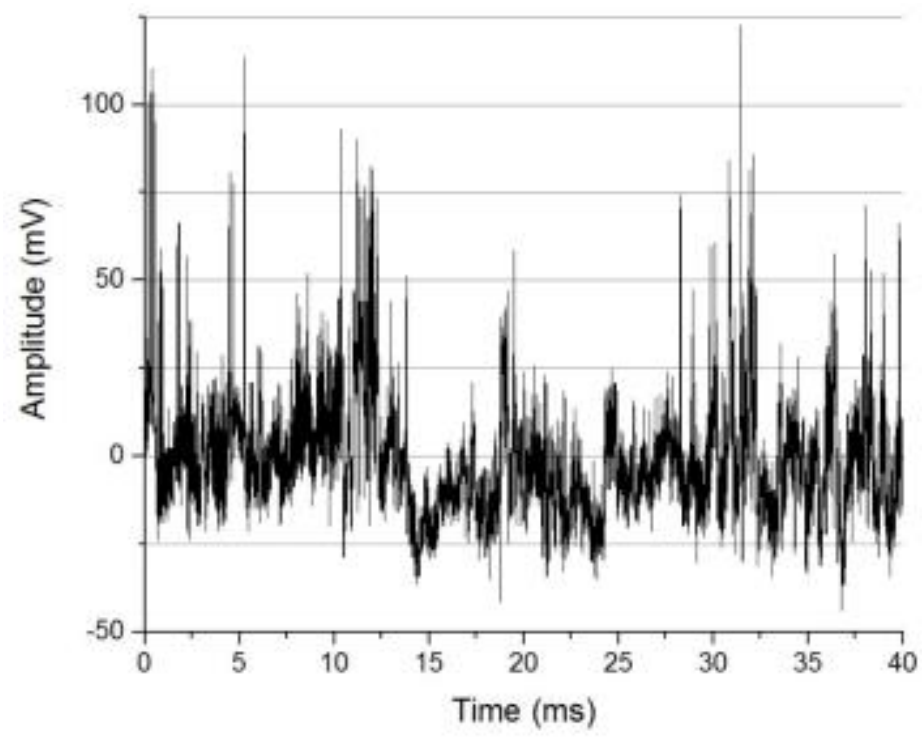

Fig. 9. Random triggered action potential recording 


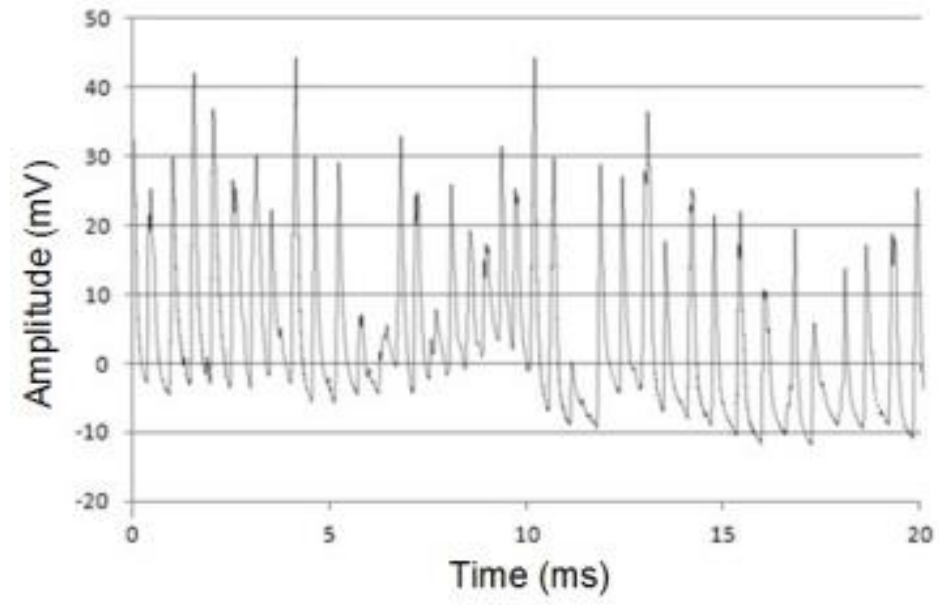

Fig. 10. The recorded extracellular potentials to $100 \mu \mathrm{M}$ of picrotoxin stimuli 


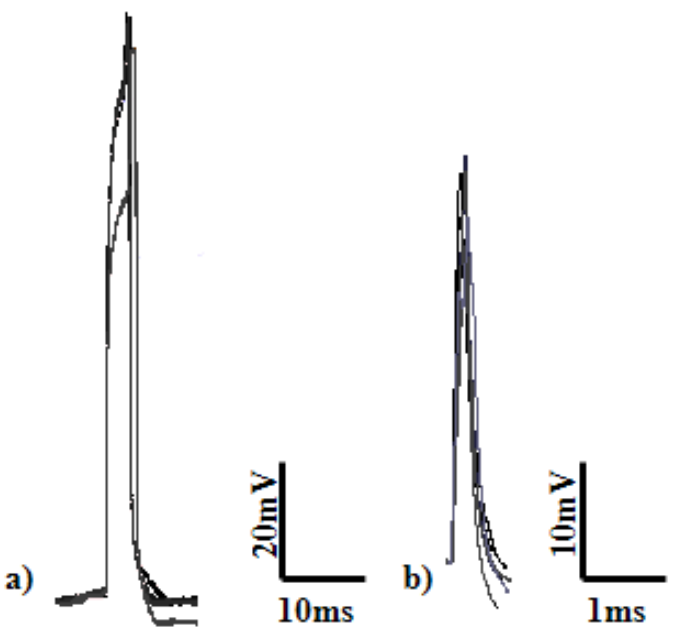

Fig. 11. "Spike type" complete waveform of recorded neuron potentials a) without and b) with $100 \mu \mathrm{M}$ of picrotoxin 